\title{
Bacterial Ecology of Otorrhea at the Laquintinie Hospital in Douala, Cameroon
}

\author{
Jean Teghonong ${ }^{1,2, *}$, Dieudonne Adiogo ${ }^{1}$ \\ ${ }^{1}$ Department of Biological Sciences, Douala University, Douala, Cameroon \\ ${ }^{2}$ Diagmed Laboratory, Douala, Cameroon \\ Email address: \\ jeanteghonong@yahoo.fr (J. Teghonong),d_adiogo@yahoo.fr (D. Adiogo) \\ ${ }^{*}$ Corresponding author
}

\section{To cite this article:}

Jean Teghonong, Dieudonne Adiogo. Bacterial Ecology of Otorrhea at the Laquintinie Hospital in Douala, Cameroon. European Journal of Clinical and Biomedical Sciences. Vol. 7, No. 4, 2021, pp. 60-64. doi: 10.11648/j.ejcbs.20210704.12

Received: July 16, 2021; Accepted: July 27, 2021; Published: August 6, 2021

\begin{abstract}
Background: Otitis media is an inflammation of the lining of the middle ear, involving the eardrum, ancillary cavities and the auditory tube. Prolonged, it can be suppurative. The etiology can be bacterial, fungal or viral. The observation of this disease led us to do this study to look for possible treatment alternatives. Method: We conducted a prospective, descriptive and cross-sectional study, from February 1 to May 3, 2013 at Laquintinie hospital in Douala. The samples taken were cultured, the identification of the strains made by the galleries API (20E TM, STAPH, 20 STREP), the study of the sensitivity to antibiotics and antifungals by the method of diffusion of discs on agar. Results: $38.3 \%$ of patients had infected left ear, $53.2 \%$ right ear and $8.5 \%$ had bilateral otitis. In $50.5 \%$ of pus, at least 2 germs were isolated. $95.7 \%$ of samples were culture positive and $4.3 \%$ negative. $79.1 \%$ of bacteria were isolated against $20.9 \%$ of fungals. Pseudomonas aeruginosa was mostly isolated $(50.9 \%)$ followed by staphylococcus aureus $(15.1 \%)$. We had better susceptibility of Pseudomonas aeruginosa to imipenem (88.5\%), piperacillin $(80.8 \%)$, ciprofloxacin $(76.2 \%)$ and significant resistance to ticarcillin $(7.7 \%)$. The best sensitivity of Candida is that of ketoconazole $(92.9 \%)$. Conclusion: The bacterial profile is different from that encountered in most Western countries. The main etiologies are: Pseudomonas aeruginosa and Staphylococcus aureus. Fungals were also present. Imipenem and ketoconazole are the antibiotic and antifungal of choice, respectively.
\end{abstract}

Keywords: Otitis, Pseudomonas Aeruginosa, Fungal

\section{Introduction}

Infections remain a major concern in various hospital services, especially otorhinolaryngology. Otitis media is an inflammation of the lining of the middle ear, involving the eardrum, ancillary cavities and the auditory tube. Prolonged, it can be characterized by discharge from the ear through a perforated tympanic membrane.

The purulent appearance, frequent, testifies to an infectious process at the level of the external acoustic meatus or the middle ear (otitis externa or otitis media of acute evolution for less than 3 weeks; subacute between 3 and 6 weeks or chronic evolving for more than 6 weeks) [1-3] the etiology can be bacterial, fungal or viral. Its management depends on the pathology in question and the responsible germ and the absence of management can lead to many serious complications [4]: extracranial (such as acute mastoiditis, facial paralysis, petrosites, labyrinthitis) and endocranial (such as meningitis, subdural or intracerebral abscesses, otogenic thrombophlebitis).

According to the studies carried out, the main causes of ear infections in Europe and more specifically in France are Haemophilus influenzae, Streptococcus pneumoniae [5, 6]. A study carried out at the Dakar University Hospital rather reveals a predominance of Staphylococcus aureus, Pseudomonas aeruginosa and Proteus [7].

The aim of our study was to find the profile of the germs responsible for purulent ear infections in Douala, Cameroon and more precisely at the laquintinie hospital in Douala and their susceptibility to various antibiotics. 


\section{Materials and Methods}

\subsection{Location and Type of Study}

This is a prospective, descriptive and cross-sectional study that was carried out at the Laquintinie hospital in Douala in the ORL (Otorhinolaryngology) services and at the Central Laboratory for a period of 03 months (from February 1 to May 3, 2013) including all patients received in ORL consultation with otorrhea (serous, mucous or purulent) in whom a clinical examination has been carried out by an ORL specialist in order to identify the disease in question and who have given their informed consent to participate in the study. The confidentiality of their data was also respected.

\subsection{Methods}

The ear sample was taken using two fine swabs (alginate or dacron mounted on a metal rod) or by aspiration of the pus after cleaning the external auditory canal with sterile physiological water. The two swabs were sent to the central laboratory in less than 15 minutes. If culturing wasn't immediately done. The use of a transport medium for bacteria was necessary.

The first swab was used to spread onto slides for direct microscopic examination and the second swab was intended for cultivation.

Macroscopic examination allowed us to know the organoleptic characteristics of the samples: smell, appearance and color.

The microscopic examination, after it's fresh state and Gram stain, could provide an interesting orientation diagnosis to communicate quickly to the clinician.

The inoculation was done by tight streaks at the start and then released using the swab or platinum loop previously sterilized in the following media: Fresh blood agar + ANC (Nalidixic acid and colistin), Chocolate agar + polyvitex, Mac Conkey, Chapman, Sabouraud agar + Chloramphenicol, Sabouraud agar + Chloramphenicol + actidione.

The fresh blood and chocolate agars were incubated in the candle jar (oxygen-poor atmosphere) at $37^{\circ} \mathrm{C}$, the Mac conkey, Chapman in the incubator at $37^{\circ} \mathrm{C}$ and the
Sabourauds in the incubator at $30^{\circ} \mathrm{C}$. The strain was identified with API galleries (20 E, 20 Strep, Staph) from BioMérieux and the antibiogram was performed according to the recommendations of CASFM / EUCAST (Antibiogramme Committee of the French Society of Microbiology / European committee on Antimicrobial Susceptibility Testing) of 2013, The antifongigram was carried out on sabouraud with chloramphenicol.

\subsection{Statistical Analysis}

The sensitivity to antibiotics and the frequency of isolated strains were assessed by calculating the proportions represented by each antibiotic and the confirmation of the hypotheses by Pearson's x2 test with Excel software or EPI info version 3.3

\section{Results}

During this study, we included 47 people and for each participant, only one sample was taken. That is to say a total of 47 samples. Our study population was comprised of $53.2 \%$ female and $46.8 \%$ male, giving a sex ratio $(\mathrm{F} / \mathrm{M})$ of 1.1

The ages of the participants ranged from 1 to 81 years with an average of $(30.2 \pm 2.3)$ years. $75 \%$ of subjects were under 40 years old.

$38.3 \%$ of patients had infected left ear, $53.2 \%$ right ear and $8.5 \%$ had bilateral otitis. We note that $95.7 \%$ of the patients came from outpatient consultations and $4.3 \%$ were hospitalized.

Table 1. Distribution of the population according to the duration of otitis.

\begin{tabular}{lll}
\hline Duration of otitis & Number & Frequency \\
\hline$<1$ week & 5 & $11 \%$ \\
1 week to 4 weeks & 16 & $34 \%$ \\
1 month to 12 months & 16 & $34 \%$ \\
2 years - 10 years & 6 & $13 \%$ \\
$>$ 10 years & 4 & $8 \%$ \\
Total & 47 & $100 \%$ \\
\hline
\end{tabular}

$8 \%$ of patients told us they had suffered from otitis for more than 10 years.

Table 2. Number of germs isolated per culture carried out.

\begin{tabular}{lll}
\hline Number of germs isolated & Number of cultures carried out & Frequency \\
\hline No germ isolated & 2 & $4.4 \%$ \\
1 germ isolated & 21 & $45.0 \%$ \\
2 germs isolated & 21 & $45.0 \%$ \\
3 germs isolated & 3 & $5.5 \%$ \\
Total & 47 & $100.0 \%$ \\
\hline
\end{tabular}

In $50.5 \%$ of pus, we isolated at least 2 germs. Out of the 47 pus samples analyzed, $95.7 \%$ of the cultures were positive and $4.3 \%$ negative.

Table 3. Distribution by group of isolated germs.

\begin{tabular}{lll}
\hline Group of pathogens & Number of germs & Frequency \\
\hline Fungal & 14 & $20.90 \%$ \\
Bacteria & 53 & $79.10 \%$ \\
TOTAL & 67 & $100.00 \%$ \\
\hline
\end{tabular}


$79.1 \%$ of bacteria were isolated against $20.9 \%$ of fungals. The number of fungals in relation to patients is $14 / 47$; or $29.79 \%$. $78.43 \%$ of fungals isolated are Candida albicans and $21.57 \%$ Candida species.

Table 4. Distribution by families and species of isolated bacteria.

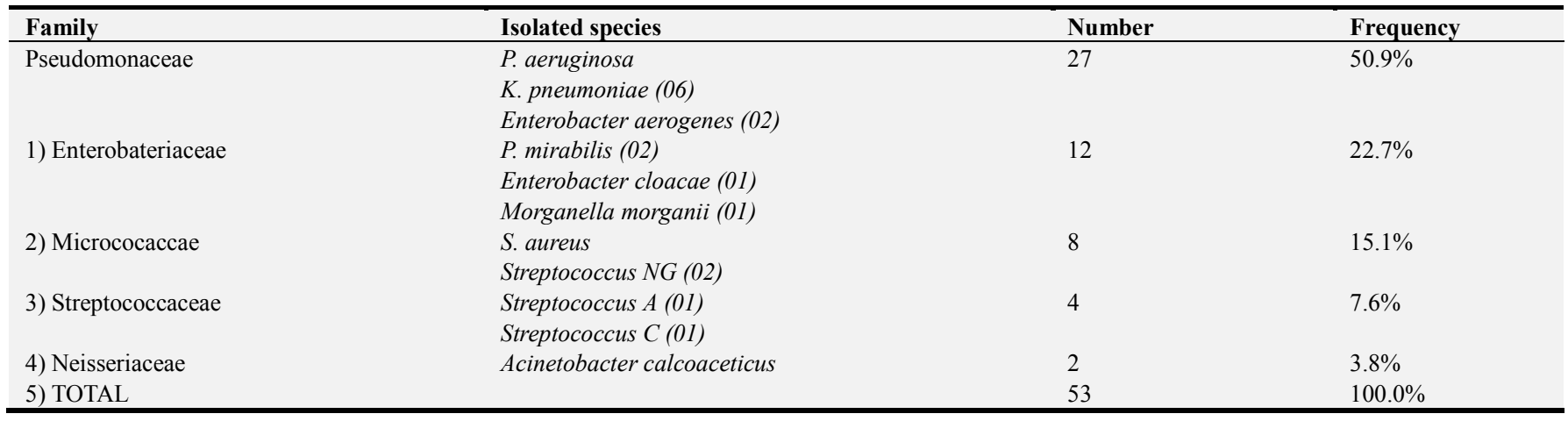

41/53 were Gram (-) and 12/53 were Gram (+).

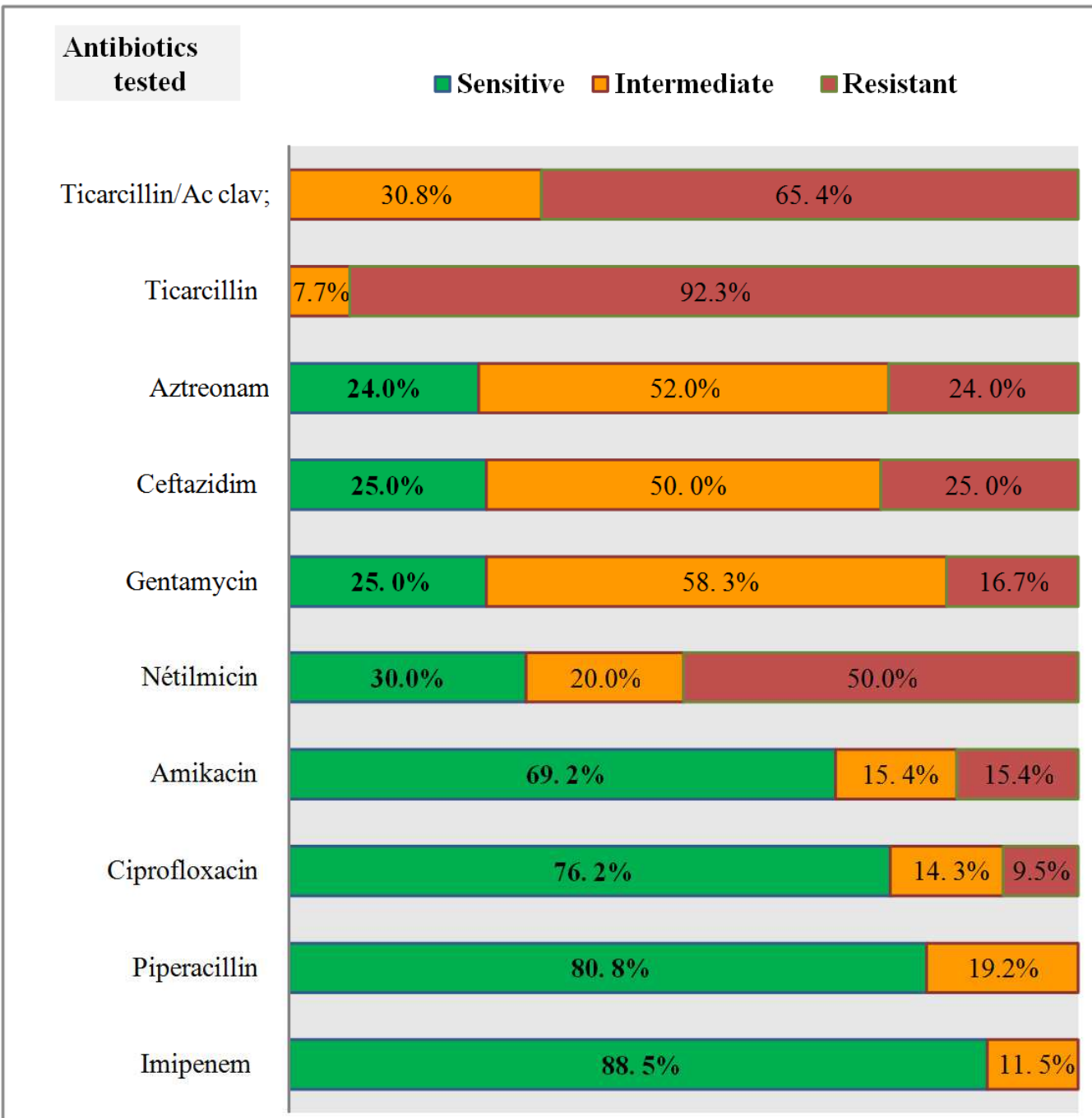

Susceptibility profile of the 27 strains of Pseudomonas aeruginosa isolated

Figure 1. Profile of Sensitivity of Pseudomonas aeruginosa to antibiotics.

We noted better sensitivity of Pseudomonas aeruginosa to imipenem (88.5\%), piperacillin (80.8\%), ciprofloxacin (76.2\%) and significant resistance to ticarcillin $(7.7 \%)$ 


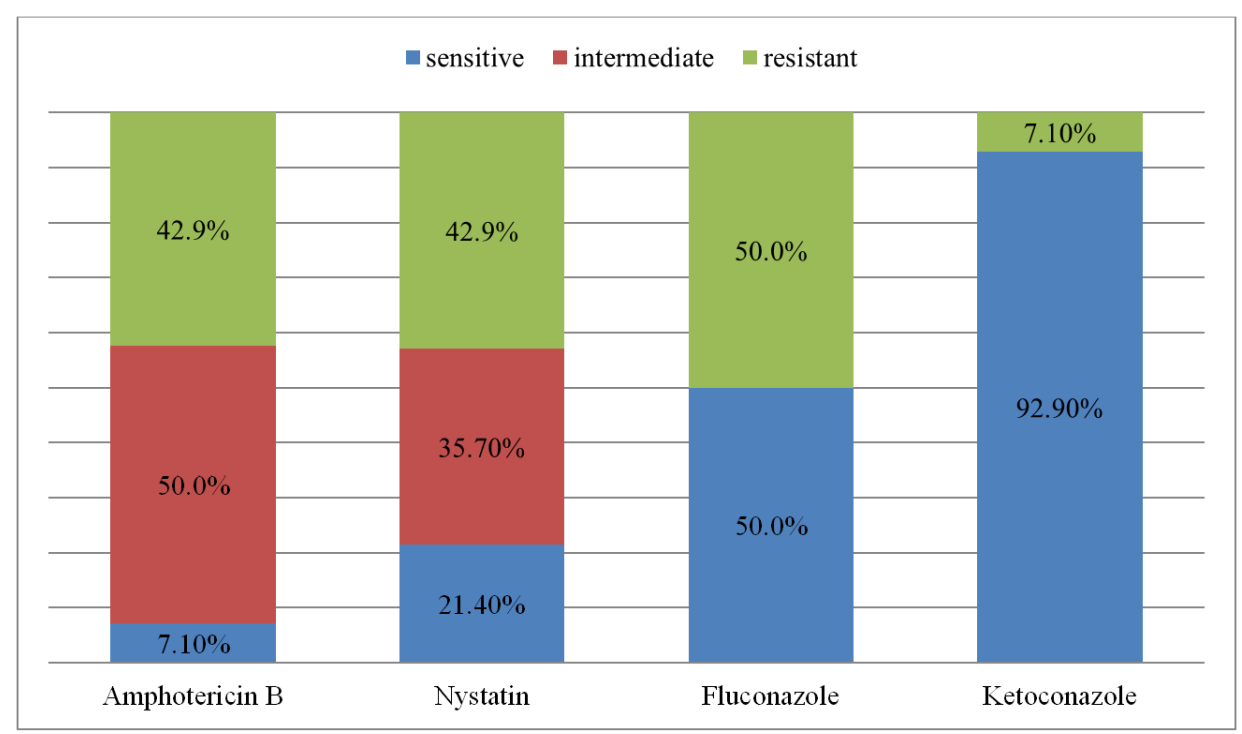

Figure 2. Profile of sensitivity of Candida to antifungals.

Susceptibility profile of the 14 strains of candida isolated to antifungals.

The best sensitivity is ketoconazole $(92.9 \%)$, followed by fluconazole $(50.0 \%)$.

\section{Discussion}

The participants were $53.2 \%$ female and $46.8 \%$ male, the same tendency was observed in studies done in Mali and Iraq $[4,11]$ contrary to studies where male was predominant $[3,8$, $9,12]$.

$53.2 \%$ of patients had otorrhea of the right ear, this is contrary to the $51.76 \%$ and $66.7 \%$ of otorrhea of the left ear found respectively by Chirwa in Malawi and Njifou in Cameroon [9, 17]. We also had bilateral otorrhea of $8.5 \%$, higher rates of $18.2 \%$ and $21.8 \%$ were reported in studies done in the same hospital where we did this research [3, 17].

We had $4.4 \%$ sterile culture, a frequency similar to the $4.8 \%$ found in Ethiopia [12], 5.26\% and 5.3\% found respectively in Mali and Nigeria [4, 8]. This can be explained by the loss of growth traits by the bacteria or otorrhea of nonbacterial origin.

We have had patients who suffered from ear infections for more than 10 years, this was also observed by Punia in North India [20], this situation would be due to a multi bacterial resistance, complications or a permanent recurrence of the infection.

We had samples with several bacteria $(50.5 \%)$ and that could be explained by the passage of inflammation from the acute phase to the chronic phase.

$20.9 \%$ of fungals were isolated, similar to $18.1 \%$ isolated in Malawi but with $10.3 \%$ of aspergillus [9]; lower rates of $2.17 \% ; 2.2 \%$ and $2.7 \%$ of fungi were found in Mali, Angola and Ethiopia respectively [4, 13, 18]. No fungal has been isolated in several studies $[8,10,14]$.

Pseudomonas aeruginosa was the predominantly (50.9\%) isolated bacterium followed by Staphylococcus aureus. Kazeem, Al-Ani and Hailu also found in first place
Pseudomonas aeruginosa (that is in Nigeria, Iraq and Ethiopia respectively). Pseudomonas aeruginosa at a respective frequency rate of $31.5 \% ; 57.5 \%$ and $30.4 \%$, followed by staphylococcus aureus $[8,11,18]$. In addition, Staphylococus aureus was mainly isolated in Mali, Bangladesh, India, southwest Ethiopia, Shanghai in China [4, $10,14,15,19]$.

We had not isolated Escherichia coli, as well as in studies $[4,19]$ but it had been isolated in Douala (Cameroon) at $7.6 \%$ [3], in Nigeria at $13.7 \%$ [8], Malawi at $8.4 \%$ [9], in Bangladesh at $13.7 \%$ [10], in Iraq at $2.7 \%$ [11], in Angola at $2.0 \%$ [13], in Ethiopia at 2, 4\% [18].

There is thus a difference in bacterial profile between most Western countries $[5,6]$ and African countries. This could be explained by the fact that the majority of ear infections in African countries are recurrent ear infections; this is due to the habits of practitioners (probabilistic management). The precarious level of hygiene in some of our hospitals in Africa favors Pseudomonas aeruginosa, which is a nosocomial bacterium. In addition Pseudomonas aeruginosa has a more adaptive capacity than other germs.

The isolation of Escherichia coli (which is a much more fecal bacteria) in some studies may be due to manual infection.

The best susceptibilities of $\mathrm{P}$. aeruginosa are: imipenem (88.5\%), piperacillin (80.8\%), ciprofloxacin $(76.2 \%)$. This is in agreement with Al-ani who found imipenem (93.8\%), ciprofloxacin (81.5\%) in Iraq [11] and Hailu in Ethiopia with ciprofloxacin $(92.0 \%)$

Our study gives a sensitivity to Ketoconazole of $92.9 \%$, fluconazole $(50 \%)$; a study carried out in Japan also gave better sensitivity to ketoconazole [16].

\section{Conclusion}

The bacterial ecology of suppurative otitis media in Cameroon is different from that of most Western countries, this requires the establishment of a therapeutic process 
adapted to our profile in order to avoid the emergence of resistant strains. As well as the occurrence of complications.

The main etiologies are Pseudomonas aeruginosa and Staphylococcus aureus. Fungals are among the germs to consider in the management of otorrhea.

Imipenem and ketoconazole are the antibiotic and antifungal of choice, respectively; Amoxicillin represents remarkable resistance to Enterobacteriaceae.

\section{Contributions of the Authors}

JT, DA participated in the design, analysis and interpretation of the data. JT to data collection and manuscript design. All authors contributed to the review and approved the final version.

\section{Conflicts of Interest}

All the authors do not have any possible conflicts of interest.

\section{Acknowledgements}

The authors kindly thank the director of the Laquintinie hospital of Cameroon and the staff of the Department of Microbiology Laboratory and ORL service for participating in sample collection. We also acknowledge Tsaplong doumbe leslie, Tonleu steeve, Danchi Henriette, Pepouna marthe from DIAGMED laboratory for their support.

\section{References}

[1] Teghonong J, Okalla Ebongue C, Yadufashije C, Kojom L, Adiogo A. Phenotypic Characteristics of Klebsiella pneumoniae Extended Spectrum $\beta$-Lactamases Producers Isolated in Hospitals in the Littoral Region, Cameroon, European Journal of Clinical and Biomedical Sciences. Vol. 6, No. 1, 2020, pp. 9-13. doi: 10.11648/j.ejcbs.20200601.13.

[2] Tran Ba Huy P. Chronic otitis media. Elementary history and clinical forms. EMC (Elsevier Masson SAS, Paris), OtorhinoLaryngology, 20-095-A-10, 2005: 25p.

[3] Njifou NA, Nadeu CO, Nsom PP, Mbanyamsig-Ndam ASR, Essama-Eno BL, Njock LR. Clinical Characteristics and Etiologies of Otorrhea at Laquintinie Hospital in Douala. Health Sci. Say: Vol 21 (1) January 2020.

[4] Sacko HB, Dembélé RK, Diallo AO, Coulibaly MS, Telly N. Bacteriology of chronic suppurative otitis media in children in Mali. J. TUN ENT - N³1 JANUARY - JUNE 2014.

[5] Robert Cohen. Otitis-conjunctivitis syndrome in 2007. Therapeutic medicine / Pediatrics. 2007; 10 (3): 172-174. doi: 10.1684 / mtp.2007.0105.

[6] Polonovsk JM. Ear infections in adults and children. the Practitioner Review. 2018; 68 (1); e15-24.

[7] Tanon-Anoh MJ, Sanogo D, Kouassi M, Folquet-Amorissani M, Kacou-N'douba A, Ette-Akre E and al. Acute otitis media in children in Abidjan: Bacteriological news 2006, vol. 53, no 3, pp. 176-181 [6 page (s) (article)] (15 ref.)].
[8] Kazeem MJ, Aiyeleso R. Current bacteriological profile of chronic suppurative otitis media in a tertiary facility of Northern Nigeria. Indian J Otol 2016; 22: 157-61.

[9] Chirwa M, Mulwafu W, Aswani JM, Masinde PW, Mkakosya R, Soko D. Microbiology of chronic suppurative otitis media at Queen Elizabeth Central Hospital, Blantyre, Malawi: A cross-sectional descriptive study. Malawi Med J. 2015; 27 (4): 120-124.

[10] Khatun MR, Alam KMF, Naznin M, Salam MA. Microbiology of Chronic Suppurative Otitis Media: An update from a Tertiary Care Hospital in Bangladesh. Pak J Med Sci. 2021; 37 (3): 821-826. doi: 10.12669/pjms.37.3.3942.

[11] Al-Ani RM, Al-Zubaidi MI, Lafi SA. Profile of aerobic bacteria and their antibiotic sensitivity in chronic suppurative otitis media in Al-Ramadi Teaching Hospital, Ramadi City, Iraq. Qatar Med J. 2021; 2021 (1): 3. Published 2021 Apr 5. doi: $10.5339 / \mathrm{qmj} .2021 .3$.

[12] Molla R, Tiruneh M, Abebe W, Moges F. Bacterial profile and antimicrobial susceptibility patterns in chronic suppurative otitis media at the University of Gondar Comprehensive Specialized Hospital, Northwest Ethiopia. BMC Res Notes. 2019; 12 (1): 414. Published 2019 Jul 15. doi: 1 0.1186/s13104-019-4452-4.

[13] Uddén F, Filipe M, Reimer $\AA$, et al. Aerobic bacteria associated with chronic suppurative otitis media in Angola. Infect Dis Poverty. 2018; 7 (1): 42. Published 2018 May 3. doi: 10.1186/s40249-018-0422-7.

[14] Chandra Sahu M, Swain SK. Surveillance of antibiotic sensitivity pattern in chronic suppurative otitis media of an Indian teaching hospital. World $J$ Otorhinolaryngol Head Neck Surg. 2019; 5 (2): 88-94. Published 2019 Feb 16. doi: 0.1016/j.wjorl.2018.05.008.

[15] Gorems K, Beyene G, Berhane M, Mekonnen Z. Antimicrobial susceptibility patterns of bacteria isolated from patients with ear discharge in Jimma Town, Southwest, Ethiopia. BMC Ear Nose Throat Disord. 2018; 18: 17. Published 2018 Dec 4. doi: 10.1186/s12901-018-0065-0.

[16] Shinogami M, IwamuraW H, Nakanishi, Yamada C. The relation of the contraction period and the bacteria of the otorrhea of chronic otitis mediaVolume 14, Supplement 1, Page 54, March 2010.

[17] Njifou A, Nsom P, Vodouhe U, Mpessa E, Moho A, Kuiffo C and al. Media acute Otitis in Douala: Epidemiological, clinical and therapeutic aspects of 120 cases. Health Sci. Dis. 2019; 20 (1): $82-6$.

[18] Hailu D, Mekonnen D, Derbie A, Mulu W, Abera BPathogenic bacteria profile and antimicrobial susceptibility patterns of ear infection at Bahir Dar Regional Health Research Laboratory Center, Ethiopia. Springerplus 2016; 5: 466.

[19] Xu J, Du Q, Shu Y, Ji J, Dai C. Bacteriological Profile of Chronic Suppurative Otitis Media and Antibiotic Susceptibility in a Tertiary Care Hospital in Shanghai, China. Ear Nose Throat J. 2020 Apr 30: 145561320923823. doi: $10.1177 / 0145561320923823$. Epub ahead of print. PMID: 32352873 .

[20] Punia RS, Singhal SK, Kundu R, Das A, Chander J. Fungal Suppurative Otitis Media (Histopathology) Among Patients in North India. Head Neck Pathol. 2019; 13 (2): 149-153. doi: 10.1007/s12105-018-0918-2. 